\title{
PRESCRIBING A RICCI TENSOR IN A CONFORMAL CLASS OF RIEMANNIAN METRICS
}

\author{
XINGWANG XU
}

(Communicated by Jonathan M. Rosenberg)

\begin{abstract}
We show that two pointwise conformal metrics that have the same
\end{abstract} Ricci tensor must be homothetic.

\section{INTRODUCTION}

The purpose of this short note is to prove the following

Theorem. Suppose $(M, g)$ is a compact, oriented Riemannian manifold without boundary of dimension $\geq 2$. If $\hat{g}=e^{2 \varphi} g$ and $\operatorname{Ric}(\hat{g})=\operatorname{Ric}(g)$, then $\varphi$ is constant. In other words, two pointwise conformal metrics that have the same Ricci tensor must be homothetic.

For the prescribed Ricci tensor equation, D. DeTurck [D1] proved the local existence theorem for a nonsingular Ricci candidate $R$. DeTurck and Koiso [DK] have shown that for every positive definite tensor $R$ on a compact manifold $M$ without boundary, there is a constant $C_{0}(R)$ with the property that, if $C>C_{0}$, then $C R$ is not the Ricci tensor of any Riemannian metric on $M$. Cao [C] recently proved that if a spherically symmetric 2-tensor $R$ is nonsingular on an $n$-sphere $S^{n}$ or an $n$-disk $B_{\delta}^{n}$, then there is at most one global spherically symmetric metric $g$ with $\operatorname{Ric}(g)=R$ up to a constant factor. As we will point out, Cao's result is just a special case of our theorem. The method we will use is totally different from Cao's, but the arguments are equally simple. We refer the reader to Cao's introduction for related facts and his important contribution to the solution of this problem.

\section{A LEMMA}

Let $(M, g)$ be a Riemannian manifold. The Ricci tensor $\operatorname{Ric}(g)$ is defined in local coordinates as

$$
R_{i j} \equiv \frac{\partial \Gamma_{i j}^{s}}{\partial x^{s}}-\frac{\partial \Gamma_{i s}^{s}}{\partial x^{j}}+\Gamma_{i j}^{s} \cdot \Gamma_{s t}^{t}-\Gamma_{i t}^{s} \cdot \Gamma_{s j}^{t}
$$

Received by the editors September 24, 1990 and, in revised form, November 28, 1990.

1980 Mathematics Subject Classification (1985 Revision). Primary 53A53, 53C20, 35G20.

Key words and phrases. Ricci tensor, conformal class, sectional curvature, spherically symmetric tensor, uniqueness. 
where

$$
\Gamma_{j k}^{i}=\frac{1}{2} g^{i s}\left[\frac{\partial g_{j s}}{\partial x^{k}}+\frac{\partial g_{s k}}{\partial x^{j}}-\frac{\partial g_{j k}}{\partial x^{s}}\right]
$$

are the Christoffel symbols of the metric $g$. If $g$ and $\hat{g}$ are two pointwise conformally equivalent metrics, then we have

Lemma. If $\hat{g}=g / u^{2}$ and $\operatorname{Ric}(\hat{g})=\operatorname{Ric}(g)$, then

$$
\operatorname{Hess}(u)=\frac{\Delta u}{n} g
$$

and

$$
(n-2) \operatorname{Hess}(u)-\left(\Delta u-(n-1) \frac{|\nabla u|^{2}}{u} g\right)=0 .
$$

Proof. By direct computation, we have the following standard formula [A, B, SY]:

(3) $\widehat{R}_{i j}=R_{i j}-(n-2) \operatorname{Hess}(\varphi)_{i j}+(n-2) \nabla_{i} \varphi \nabla_{j} \varphi-\left(\Delta \varphi+(n-2)|\nabla \varphi|^{2}\right) g_{i j}$, where $\varphi=-\log u$.

If we define $B_{i j}=R_{i j}-\left(g^{i j} R_{i j} / n\right) g$ and $S=g^{i j} R_{i j}$, then, on the one hand, we have

$$
\begin{aligned}
\widehat{B}_{i j} & =\widehat{R}_{i j}-\frac{1}{n} \hat{g}^{l k} \widehat{R}_{l k} \hat{g}_{i j} \\
& =R_{i j}-\frac{1}{n}\left[e^{-2 \varphi} g^{l k} R_{l k}\right] e^{2 \varphi} g_{i j} \\
& =R_{i j}-\frac{1}{n}\left[g^{l k} R_{l k}\right] g_{i j}=B_{i j}
\end{aligned}
$$

On the other hand,

$$
\begin{aligned}
\widehat{B}_{i j}= & \widehat{R}_{i j}-\frac{1}{n}\left[\hat{g}^{i j} \widehat{R}_{i j}\right] \hat{g}_{i j} \\
= & R_{i j}-(n-2) \operatorname{Hess}(\varphi)_{i j}+(n-2) \nabla_{i} \varphi \nabla_{j} \varphi \\
& -\left(\Delta \varphi+(n-2)|\nabla \varphi|^{2}\right) g_{i j} \\
& -\frac{1}{n} e^{-2 \varphi}\left[S-2(n-1) \Delta \varphi-(n-1)(n-2)|\nabla \varphi|^{2}\right] e^{2 \varphi} g_{i j} \\
= & B_{i j}-(n-2) \operatorname{Hess}(\varphi)_{i j}+(n-2) \nabla_{i} \varphi \nabla_{j} \varphi \\
& -\left[\left(1-\frac{2(n-1)}{n}\right) \Delta \varphi+(n-2)\left(1-\frac{n-1}{n}\right)|\nabla \varphi|^{2}\right] g_{i j} \\
= & B_{i j}-(n-2)\left[\operatorname{Hess}(\varphi)_{i j}-\nabla_{i} \varphi \nabla_{j} \varphi-\frac{1}{n}\left(\Delta \varphi-|\nabla \varphi|^{2}\right) g_{i j}\right] .
\end{aligned}
$$

Combining these two, if $n \geq 3$, we have

$$
\operatorname{Hess}(\varphi)-d \varphi \otimes d \varphi-\frac{1}{n}\left(\Delta \varphi-|d \varphi|^{2}\right) g=0 .
$$

Since $u=e^{-\varphi}$,

$$
\nabla_{i} u=-e^{-\varphi} \nabla_{i} \varphi
$$

and

$$
\nabla_{i} \nabla_{j} u=-e^{-\varphi} \nabla_{i} \nabla_{j} \varphi+e^{-\varphi} \nabla_{i} \varphi \nabla_{j} \varphi
$$


therefore,

$$
e^{\varphi} \operatorname{Hess}(u)=-(\operatorname{Hess}(\varphi)-d \varphi \otimes d \varphi)
$$

and

$$
e^{\varphi} \Delta u=-\left(\Delta \varphi-|\nabla \varphi|^{2}\right)
$$

So (4) transforms to

$$
e^{\varphi} \operatorname{Hess}(u)-\frac{1}{n}\left(e^{\varphi} \Delta u\right) g=0 .
$$

Since $e^{\varphi} \neq 0$, equation (1) follows. (3) can be written as

$$
\widehat{R}_{i j}=R_{i j}-e^{\varphi}\left[(n-2) \operatorname{Hess}(u)_{i j}-\left(\Delta u-(n-1) \frac{|\nabla u|^{2}}{u}\right) g_{i j}\right] \text {. }
$$

As a result, (2) follows from $\operatorname{Ric}(\hat{g})=\operatorname{Ric}(g)$.

\section{PROOF OF THEOREM}

Proof of Theorem. Here we assume $n \geq 3$. For $n=2$, see DeTurck's paper [D1]. Since $\widehat{R}_{i j}=R_{i j}$ and $\hat{g}=e^{2 \varphi} g$, from equation (2) we have

$$
\operatorname{Hess}(u)-\frac{1}{n-2} \Delta u g+\frac{n-1}{n-2} \frac{|\nabla u|^{2}}{u} g=0 \text {. }
$$

Therefore, if we use equation (1), equation (5) can be written as

$$
\frac{1}{n} \Delta u g_{i j}-\frac{1}{n-2} \Delta u g_{i j}+\frac{n-1}{n-2} \frac{|\nabla u|^{2}}{u} g_{i j}=0
$$

that is,

$$
\left(\frac{1}{n}-\frac{1}{n-2}\right)(\Delta u)+\frac{n-1}{n-2} \frac{|\nabla u|^{2}}{u}=0
$$

or

$$
-\frac{2}{n} u \Delta u+(n-1)|\nabla u|^{2}=0
$$

If we integrate equation (6) over $M$ and use integration by parts, we have

$$
\frac{n(n-1)+2}{n} \int_{M}|\nabla u|^{2}=0 .
$$

Since $n \geq 3, n(n-1)+2 \neq 0$. So $u$ is a constant, and therefore $\varphi$ is a constant. The proof is complete.

\section{CoRollaries AND COMMENTS}

Corollary 1. Suppose $R$ is a spherically symmetric 2-tensor on an $n$-sphere $S^{n}$ or an $n$-disk $B_{\delta}^{n}$. Then there is at most one global spherically symmetric metric $g$ with $\operatorname{Ric}(g)=R$ up to a scalar factor.

Proof. By Lemma 1.1 of Cao [C], any smooth spherically symmetric metric $g$ is globally conformally flat on a ball $B_{\delta}$; therefore, any two smooth spherically symmetric metrics $g$ and $\hat{g}$ must be pointwise conformally equivalent. For a sphere $S^{n}$, the conclusion follows directly from our theorem. For a disk $B_{\delta}^{n}$, since $u$ only depends on the distance $r$, we can assume that the metric $g$ has the form $d s^{2}=d r^{2}+r^{2} d \theta^{2}$, where $d \theta^{2}$ is the standard metric on 
$S^{n-1}$. By direct computation, we have $\Delta u=u_{r r}+(n-1) u_{r} / r$ and Hess $(u)=$ $u_{r r} d r^{2}+u_{r}\left(r^{2} d \theta^{2}\right) / r$. Equation (1) tells us that $\Delta u=n u_{r r}$ and $\Delta u / n=u_{r} / r$, hence, $r u_{r r}=u_{r}$. Using equation (6), we get

$$
u_{r}\left[(n-1) u_{r}-2 u / r\right]=0 .
$$

Differentiate this equation with respect to $r$ to get

$$
u_{r r}\left[(n-1) u_{r}-2 u / r\right]+u_{r}\left[(n-1) U_{r r}-2 u_{r} / r+2 u / r^{2}\right]=0 .
$$

Use $r u_{r r}=u_{r}$ to simplify the above equation and obtain

$$
u_{r}^{2}[2(n-2) / r]=0 \text {. }
$$

Now it is clear that $u$ must be a positive constant.

Remark 1. DeTurck asked the following question:

Question. Given a spherically symmetric 2-tensor $R$ defined on a ball or a sphere, can a spherically symmetric metric $g$ be found so that $R$ is the Ricci curvature tensor of $g$, i.e., does the Ricci system $\operatorname{Ric}(g)=R$ have a global spherically symmetric solution $g$ ? Moreover, is the solution $g$ unique if there is any?

J. Cao proved the uniqueness under the assumption that $R$ is nonsingular. We completely prove the uniqueness.

Now let $(M, g)$ be a Riemannian manifold, and let $G_{2}(M)$ denote the Grassmann bundle of 2-plane sections of $T M$ and $\pi: G_{2}(M) \rightarrow M$ the canonical projection. The sectional curvature $k$ is a real-valued funtion on $G_{2}(M)$. Let the dimension of $M$ be $\geq 3$. We say a point $p \in M$ is isotropic if $\left.k\right|_{\pi^{-1}(p)}$ is a constant; otherwise, we call $p$ nonisotropic.

Corollary 2. Suppose $(M, g)$ is an n-dimensional compact Riemannian manifold. Let $f: M \rightarrow M$ be a diffeomorphism that preserves sectional curvature. If $n \geq 3$ and the set of nonisotropic points is dense in $M$, then $f$ is an isometry. Proof. By Theorem 1 of Kulkarni [Ku], we know that the assumption on $f$ implies $f$ is a conformal diffeomorphism on the closure of the set of nonisotropic points, and by Proposition 3.1 in Kulkarni [Ku], we know that $f$ preserves the Ricci tensor; therefore, $f$ is a homothety on the closure of the set of non-isotropic points, i.e., $f$ is a global homothety. Since $f$ preserves the curvature, $f$ must be an isometry [K, p. 325].

Remark 2. If $n=3$, Kulkarni [Ku] proved the same result under the assumption that the sectional curvature of $M$ is everywhere negative. We drop this extra condition. See Theorem 6 and Theorem $7[\mathrm{Ku}]$.

Remark 3. If we relax the condition of staying in a given conformal class, then our conclusion will no longer be valid, because for Ricci flat manifolds, $R=0$ is a possible Ricci tensor and the solutions of the prescribed Ricci tensor equation form an infinite-dimensional manifold. In fact, for any diffeomorphism $f$ of $M$, if $g$ is a solution, then $f^{*} g$ is a solution, since $\operatorname{Ric}\left(f^{*} g\right)=f^{*} \operatorname{Ric}(g)=0$.

\section{ACKNOWLEDGMENT}

The author is indebted to Professor J. Cao for a preprint of his paper. He thanks Professors William Abikoff, Roger Chen, and Paul Yang for their interest in this proof, useful discussions, and helpful comments. (He also would like to thank the referee for a valuable suggestion.) 


\section{REFERENCES}

[A] T. Aubin, Nonlinear analysis on manifolds, Monge-Ampère equations, Springer-Verlag, Berlin, Heidelberg, and New York, 1982.

[B] A. Besse, Einstein manifolds, Springer-Verlag, Berlin, Heidelberg, and New York, 1987.

[C] J. Cao, On deciding which spherically symmetric 2-tensors can be Ricci Tensors, preprint, 1990.

[D] D. DeTurck, Existence of metrics with prescribed Ricci curvature, Invent. Math. 65 (1981), 179-207.

[D1] - Metrics with prescribed Ricci curvature, Seminar on Differential Geometry (S. T. Yau, ed.), Ann. of Math. Stud., vol. 102, Princeton Univ. Press, Princeton, NJ, 1982, pp. 525-537.

[DK] D. DeTurck and N. Koiso, Uniqueness and nonexistence of metrics with prescribed Ricci curvature, Ann. Inst. H. Poincaré, Anal. Nonlinéaire 1 (1984), 351-359.

[Ku] R. S. Kulkarni, Curvature and metric, Ann. of Math. (2) 91 (1970), 311-331.

[SY] R. Schoen and S. T. Yau, Riemannian geometry, Chinese Science Press, Beijing, 1988. (Chinese)

Department of Mathematics, University of Southern California, los Angeles, CA 90089-1113

E-mail address: xxu@mtha.usc.edu 